\title{
Competition for space can drive the evolution of dormancy in a temporally invariant environment
}

\author{
William H. Satterthwaite
}

Received: 26 January 2009/Accepted: 9 November 2009/Published online: 21 November 2009

(C) The Author(s) 2009. This article is published with open access at Springerlink.com

\begin{abstract}
I present a model for the evolution of a seed bank in the absence of externally driven environmental variation. I use Evolutionarily Stable Strategy (ESS) analyses of both analytic and simulation models to assess the conditions under which a dormant genotype can invade and resist invasion. In my models, plant seeds compete through lottery for discrete safe sites holding one individual each. Analyzing the conditions under which a dormant genotype can invade when rare and resist invasion once established, I conclude that dormancy can be an ESS when some fraction of seeds is retained locally, seed bank survival is high, and mortality in the seed bank is low. The advantage of dormancy stems from the ability of dormant seeds to recapture a lost site and the fact that a plant's offspring are more likely to win the lottery in its own site than in any new site. The advantage of dormancy does not depend on individual fecundity or on low relatedness with the
\end{abstract}

W. H. Satterthwaite

Department of Ecology and Evolutionary Biology,

University of California, Santa Cruz, CA 95064, USA

Present Address:

W. H. Satterthwaite $(\square)$

Applied Math and Statistics and Center for Stock

Assessment Research, University of California, Santa

Cruz, CA 95064, USA

e-mail: satterth@darwin.ucsc.edu offspring of kin, making this mechanism distinct from earlier models of sib competition.

Keywords Dormancy - Seed bank ·

Spatial competition $\cdot$ Sibling competition

\section{Introduction}

Dormant seed banks are common in nature (Leck et al. 1989). However, in light of simple evolutionary models focused on maximizing arithmetic mean growth rates this presents an apparent paradox. Seed dormancy is essentially a form of delayed reproduction, which should carry a selective penalty due to mortality and increased generation time. This problem is most often resolved by interpreting dormancy as a form of evolutionary bet hedging (Slatkin 1974), with reduced variation in yearly growth rates boosting the long-term geometric mean (Gillespie 1977). Numerous models (reviewed in Clauss and Venable 2000; Olivieri 2001; Evans and Dennehy 2005) have shown that dormancy can yield a selective advantage by reducing variance in demographic performance. Chesson and Warner (1981) also showed that dormancy could enhance the ability of an inferior competitor to resist exclusion in a varying environment. It has become virtually axiomatic that the primary explanation for seed dormancy is as a strategy for coping with variable environments. 
While some role of environmental variation in driving selection for dormancy seems indisputable, empirical tests of the relationship between environmental variability and dormancy rates have yielded mixed results (reviewed in Evans and Dennehy 2005; and see critiques of empirical study to date in Evans et al. 2007; see also Petru and Tielborger 2008). Evans and Dennehy (2005) cite Hacker and Ratcliff (1989), Ehrman and Cocks (1996), and Shem-Tov et al. (2002) as finding mixed results, and Jain (1982), Gutterman and Edine (1988), and Platenkamp (1991) as finding results contrary to the predicted positive correlation between variance in reproductive success and dormancy. In addition, Silvertown (1989) used data on annual variation in survival and fecundity to predict whether any degree of dormancy should be present in 11 annual grassland species, correctly classifying eight of them. Interestingly, however, all of the errors in his classification scheme were predictions of no dormancy in species that in fact displayed it. Doak et al. (2002) used long-term demographic data to estimate the dormancy rates that would minimize extinction risk in two short-lived plants of conservation concern, and in both cases predicted extinction risk would be minimized with no dormancy unless environments were more variable than the data suggested. Several species are able to modify their germination fraction in response to environmental cues (Baskin and Baskin 1998), but even species with reliable cues often maintain some degree of dormancy despite encountering a cue indicating a favorable environment (Philippi 1993a). The weakness of empirical support for a tight link between environmental variability and dormancy suggests that other factors may also be important in selecting for dormancy even in temporally invariant environments.

The only alternative explanation for the prevalence of dormancy that has received appreciable attention in the literature is as a means of reducing sib competition (Ellner 1986; anticipated by Venable and Lawlor 1980). If dormancy is under maternal control and related offspring are likely to occur in close proximity and compete strongly, seed dormancy may increase the mother plant's fitness even if the inclusive fitness of the offspring is maximized with no dormancy (Ellner 1986). However, empirical evidence for the importance of sibling competition in plants is lacking (Cheplick 1992), and there is even less evidence that the strength of sibling competition varies consistently with differing germination strategies. Sibling competition models predict that dormancy rates should increase with family size (Ellner 1986). Observational studies by Zammit and Zedler (1990) and experimental manipulations of plant size by Philippi (1993b) showed that larger plants produced seeds that germinated less readily, consistent with this hypothesis. However, Hyatt and Evans (1998) found only a weak relationship between family size and germination fraction in the desert mustard Lesquerella fendleri, and the study did not account for other factors that could covary with family size and germination fraction.

Besides, many modeling studies suggest sib competition should favor dormancy only under fairly restrictive conditions. Ellner's (1986) models show an advantage to dormancy only when plants produce "many" seeds, and all of these seeds reach suitable sites. Nilsson et al. (1994) suggest a strong advantage to dormancy, but they restrict their model to a single patch such that dispersal is unable to serve any role in ameliorating sibling competition, and they ignore the increased generation times resulting from dormancy. Besides, given restricted dispersal, dormant sibs will still compete with the offspring of sibs. Therefore, dormancy will not eliminate competition between closely related individuals, especially for inbred, selfing, or asexual populations (Kobayashi and Yamamura 2000), and models need to account for competition with non-sibs as well (Tielborger and Valleriani 2005). Inbreeding might be expected to be especially prevalent in plants with limited dispersal and small genetic neighborhoods, the same plants where substantial sib competition is most likely. Therefore, a model allowing selection for dormancy even in the face of close relatedness to the offspring of sibs should be more generally applicable than models that rely on the assumption that the offspring of sibs will be minimally related.

While it appears that sib competition may not drive selection for dormancy in many situations, very few alternative models have been proposed to explain the evolution of dormancy in a constant environment. Ellner (1987) and Lalonde and Roitberg (2006) suggested that dormancy might be favored as a response to cyclic or chaotic population dynamics, but there is little evidence to believe cyclic or chaotic dynamics are present in many plant populations (Rees 
and Crawley 1991). Rees' (1994) ESS analysis suggested that age structure and synchronous reproduction could select for dormancy in a system consisting of a finite number of safe sites in which plants could establish. However, this model requires a rigid synchrony of reproduction across all individuals in a population established from a single cohort. It also requires that the system be entirely limited by the availability of safe sites, assuming seeds were so numerous and well dispersed that every site was saturated by seeds, even from the rare invader.

As a non-exclusive alternative, I present a model of selection for seed dormancy in a temporally invariant environment. I assume a fixed number of safe sites and finite seed production, and assume competition between two asexual genotypes. Since all offspring are clones, individuals are equally related to sibs and the offspring of sibs. Thus, any advantage of dormancy in this scenario is robust to the effects of inbreeding and this allows me to directly address the question of whether a genotype with dormancy can invade and take over a population. I will show that some degree of dormancy is frequently an evolutionarily stable strategy (able to invade a population without dormancy, and able to resist invasion by a nondormant genotype) as long as there is some local retention of seeds. I first present an analytic model for the limiting case where all sites are occupied and dormancy lasts one generation, and show that the advantage of dormancy in this model does not depend on individual fecundity as in earlier sib-competition models. Additionally, the analytic model shows that the advantage of dormancy stems from the space-holding role of dormant seeds. In an annual plant system, site occupancy turns over each year, creating the potential for a different genotype to take over a given site. The genotype currently occupying a site gains some advantage in that it can deposit a majority of its seeds in that site, leading to a good chance of winning the lottery competition for the site the following year, but any time a genotype is replaced at a site it completely loses this home site advantage unless it has built up a seed bank over multiple years. For ease of presentation, I first present this analytic model under the assumption that the first dormant adult to establish is accompanied by dormant siblings in the seed bank, as would be the case if the dormancy mutation first arose in the germ line of a mother plant. However, I show that this assumption can be removed (at the cost of more complex model presentation) in the Appendix.

I will then present simulation models that relax the restrictive assumptions about saturation of all safe sites and only short-term dormancy. Using spatially explicit, individual-based models I will show that dormancy can still be a favored strategy when the dormant genotype is more likely to recapture a lost site than the non-dormant genotype.

\section{Analytic model: methods}

Consider a system of $N$ sites of which a proportion $s$ is safe, with $N$ a large number (variables are summarized in Table 1). Assume that all safe sites are occupied and that the fecundity of the plants is high enough that all sites are expected to receive some seed input. Each site hosts a single annual plant that produces $F$ seeds. Each seed has a probability $r$ of being retained in its parent's site and a probability $(1-r)$ of dispersing, landing in any site with equal probability. The probability of a dispersing seed landing in any given site is therefore $1 / N$. When multiple seeds are present in a site, each seed is equally likely to form the one successful colonist the site can support. Thus, if multiple genotypes are present, there is a competitive lottery with the probability of a given genotype establishing in that site equal to the number of seeds of that genotype germinating in the site divided by the total number of seeds (of all genotypes) germinating in the site.

First, consider a monoculture of plants without dormancy. For any given safe site, the seed rain into the site from the retained seed production of the resident plant is $r F$. The seed rain into any given site from the dispersed seed production of the entire population is $s N(1-r) F(1 / N)=s(1-r) F$. This represents the product of the total number of plants producing seeds, the fraction of seeds each plant disperses, and the probability each dispersing seed lands in the site of interest.

Now consider an "invasion" by a single plant, also with no dormancy, establishing as an adult in year 0 . The expected number of daughters the invader will leave in her home site is equal to her seed input into the site divided by the total seed input into the site. Ignoring the small chance that seeds that disperse out of her site might land in it with probability $1 / N$, the 
Table 1 Variables used in analytic model

\begin{tabular}{ll}
\hline$N$ & Number of sites \\
$s$ & Proportion of sites safe (habitable) \\
$F$ & Fecundity \\
$r$ & Probability a seed is retained in mother's home site \\
$g$ & Probability of germination in first year, for mutant seed \\
$b$ & Survival probability of a dormant seed \\
$E_{\mathrm{h}}$ & Expected number of descendants (probability of a descendant) \\
$E_{\mathrm{s}}$ & in a plant's original home site \\
$E_{\mathrm{n}}$ & Expected number of descendants in new sites, per seed \\
$E_{\mathrm{r}}$ & Total expected number of descendants in new sites \\
$E_{1}$ & Probability of recapturing a lost site through the seed bank \\
$E_{2}$ & Expected total number of descendants (sites occupied by invaders) in year 1 \\
\hline
\end{tabular}

expected number of daughters she leaves in her original home site is

$E_{\mathrm{h}}=\frac{r F}{r F+s(1-r) F}=\frac{r}{r+s(1-r)}=A$

The expected number of daughters the invader will leave in new sites from a single dispersing seed is the joint probability that the single dispersing seed encounters a safe site $(s)$ and that it captures the site:

$E_{\mathrm{s}}=\frac{s}{r F+s(1-r) F}$

If sites are sufficiently numerous that two of a plant's dispersing seeds are unlikely to land in the same site, the expected total number of daughters in new sites will be the product of the total number of seeds the plant disperses and the probability that each dispersing seed captures a new safe site:

$E_{n}=\frac{s(1-r) F}{r F+s(1-r) F}=\frac{s(1-r)}{r+s(1-r)}=B$

Therefore, the total expected number of daughters in year 1 is:

$$
\begin{aligned}
E_{1} & =E_{h}+E_{n}=\frac{r}{r+s(1-r)}+\frac{s(1-r)}{r+s(1-r)} \\
& =\frac{r+s(1-r)}{r+s(1-r)}=1
\end{aligned}
$$

Note that the expected number of daughters is one, as would be expected for invasion of a saturated population by an identical genotype. The expected number of granddaughters (sites occupied by invader adults in year 2) is thus obviously also one, but it is instructive to write out the terms for comparison with the expected number of descendants in year 2 for a dormant invader.

$$
\begin{aligned}
E_{2}= & E_{\mathrm{h}} E_{1}+E_{\mathrm{n}} E_{1} \\
= & \frac{r}{r+s(1-r)}\left(\frac{r}{r+s(1-r)}+\frac{s(1-r)}{r+s(1-r)}\right) \\
& +\frac{s(1-r)}{r+s(1-r)}\left(\frac{r}{r+s(1-r)}+\frac{s(1-r)}{r+s(1-r)}\right) \\
= & A(A+B)+B(A+B) \\
= & \frac{r}{r+s(1-r)}(1)+\frac{s(1-r)}{r+s(1-r)}(1)=1
\end{aligned}
$$

Now consider an invader that has dormancy. Seeds either germinate immediately with probability $g$, or they survive for 1 year in the seed bank with probability $b$ and the remaining fraction $(1-g) b$ of the seeds germinate the next year. I will begin modeling from the standpoint of a single-established adult invader accompanied by dormant siblings in the seed bank (all present in year 0), as might be expected if the mutation giving rise to dormancy occurred in the germ line of a parent plant prior to year 0 . I will project forward for two generations and calculate the expected number of descendants. I will ignore seeds still in the seed bank two generations in the future, making this a conservative test of the advantage of dormancy. Following Hamilton (1967), I will compare the expected number of descendants 2 years in the future (comparable to granddaughters as above, but the term granddaughter is not strictly appropriate due to time lags introduced by dormancy, allowing daughters and granddaughters of the same individual to co-occur), for the resident genotype and for an invader with dormancy. If the expected number 
of descendants in year 2 for the invader is greater than one, the invader should increase in frequency relative to the non-dormant resident.

This analysis neglects the possibility that the mutation yielding dormancy may have occurred in just a single seed, meaning that in year 0 of the invasion there should be only a single mutant adult with no siblings in the seed bank. In the first year of such an invasion, the expected number of offspring for the invader will be less than one since the seed bank cannot immediately contribute offspring and the total seed production in the first year is the same as the nondormant resident. However, so long as the mutant is predicted to increase in frequency when rare, it should continue to increase in frequency for multiple generations, since only the very first generation of the invasion will not benefit from the seed bank. Further, in the Appendix I show that the assumption that the first mutant adult is accompanied by mutant siblings in the seed bank is not a requirement for successful invasion.

The expected number of descendants the invader leaves in the first year is calculated as for the nondormant invader, but with recruitment from the seed bank factored in. As before, the expected number of descendants is the sum of the expected number of descendants in the home site and the expected number of descendants in new sites.

The invader's home site will receive an input $g r F$ from seeds the invader produced this year which were not dormant. In addition, it will receive $(1-g) b r F$ surviving seeds germinating out of the seed bank from seeds the invader produced (and retained) last year that were dormant. As before, plants from other sites (all of which have the nondormant genotype) will contribute $s(1-r) F$ seeds to the site. Therefore:

$$
\begin{aligned}
E_{h} & =\frac{(g+(1-g) b) r F}{(g+(1-g) b) r F+s(1-r) F} \\
& =\frac{(g+(1-g) b) r}{(g+(1-g) b) r+s(1-r)}=A^{\prime}
\end{aligned}
$$

Note that $A$ ' is very similar to $A$, but will always be slightly smaller since $g$ and $b$ are less than one.

In order to colonize new sites, the invader can capture sites either through the dispersal of freshly germinating seeds or through the germination of surviving dormant seeds it dispersed the previous year. Note that $g$ does not appear in the denominator since the resident seeds in these new sites would be produced by wild-type plants, as would almost all of the seeds dispersing in from other sites:

$$
\begin{aligned}
E_{\mathrm{n}} & =\frac{(g+(1-g) b) s(1-r) F}{r F+s(1-r) F} \\
& =\frac{(g+(1-g) b) s(1-r)}{r+s(1-r)}=B^{\prime}
\end{aligned}
$$

Note that $B$ ' is very similar to $B$, but will always be slightly smaller since $g$ and $b$ are less than one.

Calculating the expected number of descendants in year 2 for the invader requires an additional term to account for the fact that the invader might lose its home site in year 1 but recapture it in year 2 due to the seeds it left in the seed bank:

$E_{2}=E_{\mathrm{h}} E_{1}+E_{\mathrm{n}} E_{1}+\left(1-E_{\mathrm{h}}\right) E_{\mathrm{r}}$

The expected number of descendants created from a site retained in the first year is calculated as before:

$$
\begin{aligned}
E_{\mathrm{h}} E_{1}= & \frac{(g+(1-g) b) r}{(g+(1-g) b) r+s(1-r)} \\
& \times\left(\frac{(g+(1-g) b) r}{(g+(1-g) b) r+s(1-r)}\right. \\
& \left.+\frac{(g+(1-g) b) s(1-r)}{r+s(1-r)}\right)=A^{\prime}\left(A^{\prime}+B^{\prime}\right)
\end{aligned}
$$

Note that this is slightly smaller than the corresponding term in the number of descendants expected for the non-dormant invader $A(A+B)$, reflecting the cost of mortality in the seed bank.

When calculating the expected number of descendants in year 2 produced by plants in new sites the invader colonized during the first year, the contribution from dormant seeds is dropped since the dormant seeds produced by plants in sites captured just 1 year ago will not make a contribution until they germinate the next year:

$$
\begin{aligned}
E_{\mathrm{n}} E_{1}= & \frac{(g+(1-g) b) s(1-r)}{r+s(1-r)} \\
& \times\left(\frac{g r}{g r+s(1-r)}+\frac{g s(1-r)}{r+s(1-r)}\right) \\
& \text { substituting : } \\
& A^{\prime \prime}=\frac{g r}{g r+s(1-r)} \\
& B^{\prime \prime}=\frac{g s(1-r)}{r+s(1-r)} \\
& \text { yields: } \\
& =B^{\prime}\left(A^{\prime \prime}+B^{\prime \prime}\right)
\end{aligned}
$$


A final term must be added to account for the possibility that the invader may lose its home site in the first year but recapture it in the second year. The invader will have left behind $(1-g) b$ of the $r F$ seeds it retained in its site at the start of the invasion, meaning $(1-g) b r F$ seeds from the invader will germinate in a site it had lost the previous year. This site will have a total seed input of $(1-g) b r F$ seeds from the seed bank, $r F$ from the new (nondormant) occupant, and $s(1-r) F$ from seeds dispersing in from all other sites.

$$
\begin{aligned}
& \left(1-E_{\mathrm{o}}\right) E r=\left(1-\frac{(g+(1-g) b) r}{(g+(1-g) b) r+s(1-r)}\right) \\
& \quad \times \frac{(1-g) b r F}{(1-g) b r F+r F+s(1-r) F} \\
& =\left(1-\frac{(g+(1-g) b) r}{(g+(1-g) b) r+s(1-r)}\right) \\
& \quad \times \frac{(1-g) b r}{(1-g) b r+r+s(1-r)}=\left(1-A^{\prime}\right) C
\end{aligned}
$$

where

$$
C=\frac{(1-g) b r}{(1-g) b r+r+s(1-r)}
$$

As it must, this term reduces to zero if $g=1$.

Adding all the three terms together yields the expected number of descendants (sites occupied by established plants) in year 2 for the invader:

$$
\begin{aligned}
& E_{2}=\frac{(g+(1-g) b) r}{(g+(1-g) b) r+s(1-r)} \\
& \times\left(\frac{(g+(1-g) b) r}{(g+(1-g) b) r+s(1-r)}+\frac{(g+(1-g) b) s(1-r)}{r+s(1-r)}\right) \\
& +\frac{(g+(1-g) b) s(1-r)}{r+s(1-r)}\left(\frac{g r}{g r+s(1-r)}+\frac{g s(1-r)}{r+s(1-r)}\right) \\
& +\left(1-\frac{(g+(1-g) b) r}{(g+(1-g) b) r+s(1-r)}\right) \\
& \times \frac{(1-g) b r}{(1-g) b r+r+s(1-r)} \\
& =A^{\prime}\left(A^{\prime}+B^{\prime}\right)+B^{\prime}\left(A^{\prime \prime}+B^{\prime \prime}\right)+\left(1-A^{\prime}\right) C
\end{aligned}
$$

Note that if $g=1$, this reduces to the formula for the expected number of descendants for the nondormant invader. The key determinant of the success of the invader is whether the addition of the third term $\left(1-A^{\prime}\right) C$, representing the ability of dormant seeds to recapture home sites lost by the invader, is enough to boost the expected number of descendants above 1 for some value of $g<1$.

In order to establish that a dormant strategy is an ESS, it must also be able to resist invasion. Identifying criteria for the failure of an invasion by the nondormant genotype requires a consideration of multiple generations, since a given site becomes more hospitable for the non-dormant invader once it holds it for more than 1 year (long enough to deplete the locally produced seed bank). This problem is addressed fully in the Appendix.

\section{Simulation model: methods}

The primary goal of the simulation model was to identify the combinations of (1) fecundity, (2) dispersal ability, and (3) safe site availability for which some degree of (4) seed dormancy (germination rate $<1$ ) was an evolutionarily stable strategy, under less restrictive assumptions about saturation of safe sites and the length of the dormant stage. Identifying scenarios in this four-dimensional space where dormancy was favored required first determining steady-state population sizes for asexual populations of a single genotype with a fixed germination strategy. Once a steady-state population was achieved, it was invaded by a second asexual population, which was identical in all respects except for its germination rate. The details of model structure are highly similar to Satterthwaite (2007), here I summarize the workings of the model and describe key elaborations.

Single-genotype model

Modeled plants inhabited a $100 \times 100$ grid of square cells with periodic boundaries. Some fraction of these sites were safe sites, where a single plant could germinate and complete its annual lifecycle, and were arranged randomly on the grid. Each adult plant produced an identical number of seeds. Some fraction of seeds were retained in the home site, the remainder were distributed randomly among all sites. Additional simulations were performed with seeds traveling a random distance and direction from a negative exponential dispersal kernel (Satterthwaite 2004), but these simulations did not differ in their qualitative 
behavior and required much longer simulation run times and higher replication to establish patterns, so they are not presented in this article. Seeds landing in an unsafe site died. Germinating seeds landing in a safe site established plants in that site the next year, although a site would support only one adult even if colonized by multiple seeds. I projected all simulations repeatedly until the proportional difference between the 10-year running average of total aboveground plant number as determined on consecutive years differed by less than one ten-thousandth. I repeated simulations for different values of safe site availability, seed production per individual, and mean seed dispersal distance to establish initial conditions for invasion by a dormant genotype.

\section{Multiple-genotype model}

In order to determine optimal germination strategies for different fecundities, dispersal abilities, and safe site availabilities, I first ran non-dormant singlegenotype simulations until population sizes stabilized. I then randomly replaced $10 \%$ of the existing plants with plants of a different genotype and germination rate. I used this relatively large starting population size because of the demographic stochasticity introduced by random dispersal and lottery competition for sites, which otherwise often led to rapid extinction of invaders even if they were slightly more fecund than the resident. A common assumption of ESS analyses is that an invader with even a slight advantage can invade, but in stochastic projections individual invasions are likely to fail. However, if the invader has a deterministic advantage, given repeated invasions (e.g., through repeated origins of a mutant genotype) eventual success should be expected. Both genotypes reproduced asexually and were identical in all respects except for the germination rate. Germinating and newly produced dormant seeds were dispersed as described for the singlepopulation simulations. Dormant seeds reaching safe sites were added to the seed bank for that site. Each year, seeds in the seed bank germinated with a constant probability, while seeds remaining dormant either persisted in the seed bank at their site or died. For sites containing germinating seeds from plants of both genotypes, the genotype establishing in the site was chosen in a lottery competition process, with the probability of a genotype establishing in a site equal to its proportional representation in the total number of germinating seeds for that site.

In order to find an evolutionarily stable germination strategy for a given fecundity and dispersal capability, I searched for a germination fraction that could invade any other germination fraction and resist invasion by any other germination fraction once established. I considered a genotype capable of invading another if its net growth rate over a 20year invasion was positive as determined by averaging 200 replicate invasions (500 replicate invasions for Fig. 3c, to yield a smoother surface). I considered a resident genotype capable of resisting invasion if the invader's average net growth rate was negative. For dormant genotypes which were able to invade, I ran additional simulations with the dormant genotype allowed to grow to an equilibrium size and then subjected it to invasion by the nondormant genotype.

In order to test the hypothesis that the advantage of dormancy stems from its space-holding role, making it easier for a dormant genotype to recapture safe sites than a nondormant genotype, I ran repeated 100 year simulations for dormant and non-dormant genotypes competing with various combinations of fecundity and seed dispersal and initial populations of 100 plants of each genotype. The two genotypes were identical except that one genotype had an $80 \%$ germination rate and $90 \%$ seed survival, while the other had no dormancy. In year 98 of the simulation, I identified all sites occupied by each genotype and identified all the sites lost by each genotype in year 99. Tracking only these sites in year 100, I calculated the probability of each genotype recapturing a site it lost the previous year. This was a conservative test of the space-holding ability of a seed bank, since the dormant genotype might recapture a site through germination of a dormant seed more than 1 year after the site was first lost.

I let these simulations run for many years to allow a seed bank to establish. However, this allowed the favored genotype to increase in abundance more than the disfavored genotype, giving it a colonization advantage because more plants were producing and dispersing seeds of that genotype. For a more fair comparison, I repeated these simulations with the more abundant genotype reduced to equal abundance with the other genotype, randomly picking plants of that genotype to remove. When I removed plants with the dormant genotype, I also removed the seed bank 
from the corresponding site. I ran these simulations for 50 years rather than 100 to avoid the very small total population sizes that would result if the disfavored genotype were very rare by year 98 .

\section{Analytic model: results}

For an invader with seed bank survival $b$ equal to 0.9 and the proportion of safe sites $s$ equal to 0.05 , the dormant genotype can invade over a range of dispersal and germination rates (Fig. 1). The invasion never succeeds without some local retention of seeds. Invasion is most successful given intermediate values for the germination rate and dispersal fraction (although given the results in the Appendix, it appears that invaders with low germination rates cannot succeed if the initial adult invader is not accompanied by dormant siblings in the seedbank). Note that as germination approaches 1 , the number of descendants approaches 1 from above, so the graph implies that genotypes with germination rates arbitrarily close to 1 can invade. While this is the case, genotypes with intermediate germination rates are the most successful invaders. Further, for all of parameter space where the dormant genotype can invade, it can also resist invasion by the non-dormant genotype (see Appendix). Genotypes with very low germination rates that do not allow the dormant morph to invade

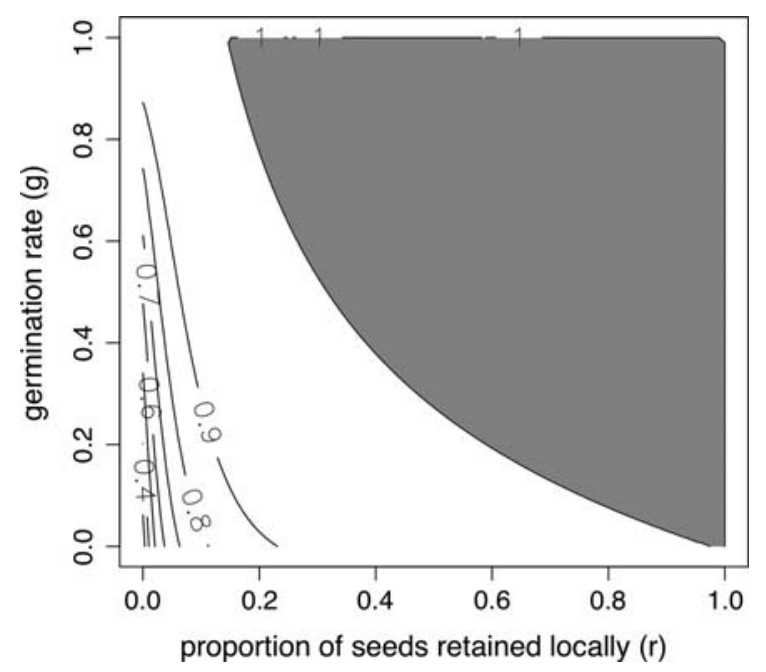

Fig. 1 The expected number of descendants in year 2 for an invading genotype given annual seed bank survival equal to 0.9 and $5 \%$ of sites safe. The shaded region indicates $>1$ descendants in year 2 and invasion success expected still do a very effective job at resisting invasion, since an invader will face competition with a very large seed bank in its home site.

We can determine the importance of seed bank survival and safe site availability in determining the fitness of the dormant strategy by comparing the area of germination-seed retention phase space over which invasion succeeds. Invasion always fails for seed bank survival less than approximately 0.8 (Fig. 2), making the point that survival in the seed bank is crucial to the success of the dormant strategy. Given adequate survival in the seed bank, invasion by a dormant genotype is easier when safe site availability is low. This is to be expected, since dormancy yields an advantage through the potential to recapture lost home sites despite a reduction in the probability of capturing new sites. As safe site availability decreases, the relative importance of retaining or recapturing the home site increases since capturing new sites becomes increasingly unlikely.

\section{Simulation model: results}

Conditions favoring dormancy

Dormancy appears to be favored under a broad range of conditions when some seeds are retained locally and

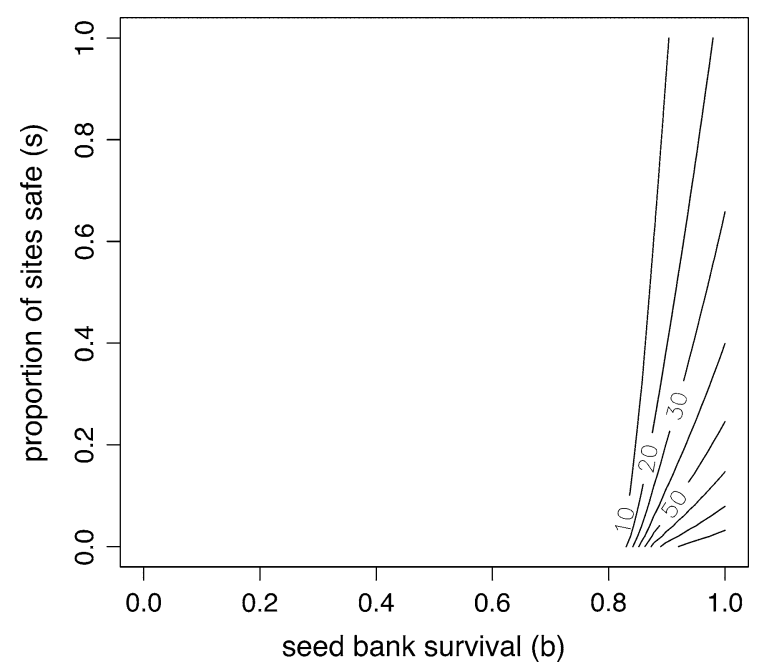

Fig. 2 The percent of germination-seed retention phase space over which the dormant genotype can invade, for different combinations of local seed retention and safe site availability 
Fig. 3 Log of 20 year geometric mean growth rate $(\mu)$ as a function of germination rate and probability of local seed retention for a dormant genotype with a fecundity of 8 seeds per plant and $90 \%$ annual survival, b 32 seeds and $90 \%$ survival, and c 8 seeds per plant and $80 \%$ annual survival. Invasions succeed if $\mu$ is positive (shaded regions). Contours were inferred using the contour command in R ( $\mathrm{R}$ Development Core Team 2007) fitted to 200 replicates (500 for Fig. 3c) for each germination-retention combination, with germination and retention varied from 0.1 to 0.9 in increments of 0.1

seed bank survival is high. When the annual survival probability of dormant seeds was 0.9 , a dormant morph could successfully invade a non-dormant morph for a wide range of local retention and germination probabilities (Fig. 3a, b) so long as local seed retention was not zero. Reducing annual seed bank survival to 0.8 made invasion by a dormant morph more difficult (Fig. 3c), but the evolutionarily stable germination strategy still often included some degree of dormancy. Consistent with the analytic model, fecundity had little effect on the parameter space for which dormancy was selected (Fig. 3a, b). As would be expected, genotypes with a very high probability of remaining dormant faired poorly, presumably losing too many seeds to mortality while still in the seed bank. Additional simulations show the advantage of some degree of dormancy also holds at much higher fecundities, at least as high as the maximum value modeled of 512 seeds per plant (data not shown).

In all the cases in which a dormant morph could invade, it could successfully resist invasion once established. In fact, dormant morphs could resist invasion over a wider range of parameter space than they could invade. This is not surprising, since the benefits of a seed bank can only be accrued after the seed bank has built up through time, so a seed bank is less advantageous in the early stages of an invasion.

However, for all simulations in which there was no local retention of seeds, dormant genotypes could never invade and could never resist invasion. This is consistent with earlier theoretical findings that dormancy should be universally disfavored in temporally invariant environments and suggests that some degree of locally restricted dispersal is key to the mechanism by which dormancy can provide a selective advantage in this model of a temporally invariant environment.

The hypothesis that dormancy provides a competitive advantage through its space-holding role received

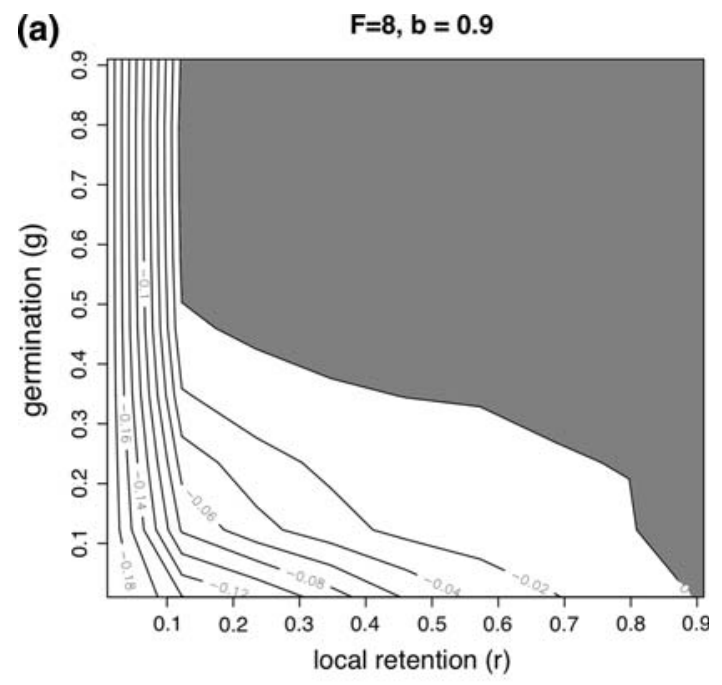

(b) $\quad F=32, b=0.9$

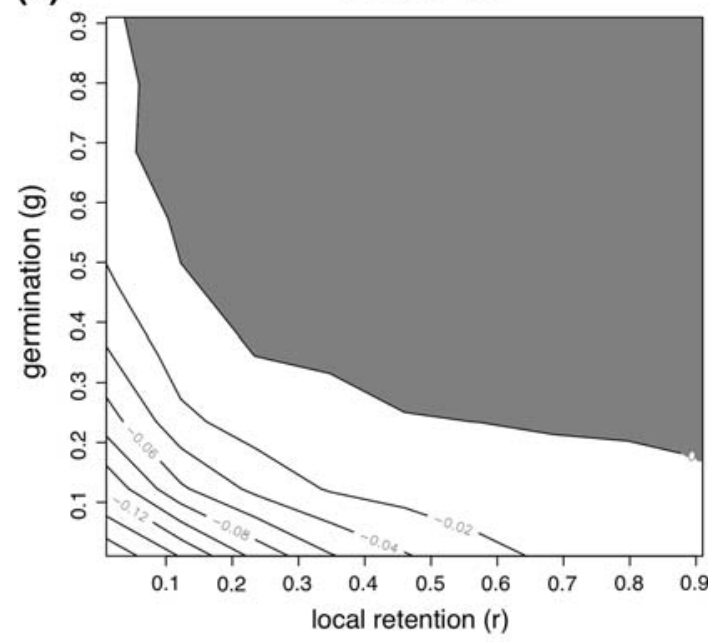

(c)

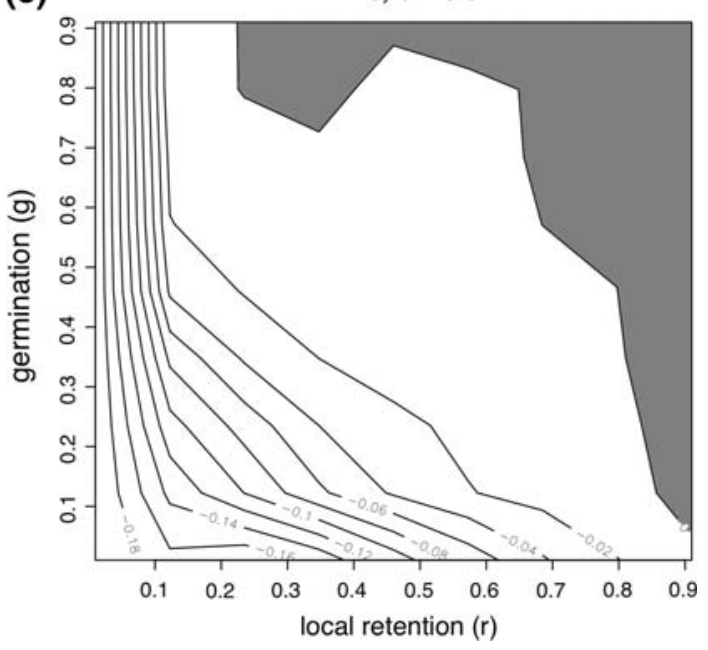


strong support, consistent with the analytic model. Tracking individual sites, dormant genotypes were more likely to recapture lost sites than non-dormant genotypes for parameter combinations where the dormant genotype could successfully invade, whereas non-dormant genotypes were more likely to recapture lost sites in scenarios where the dormant genotype could not invade. For simulations in which I did not reduce genotypes to equal abundances, the dormant genotype always had a higher probability of recapturing sites than the non-dormant genotype whenever there was some degree of local retention of seeds (Table 2). In all cases where the non-dormant genotype recaptured at least one site, $\chi^{2}$ tests showed a significantly higher proportion of sites being recaptured by the dormant morph except in cases where there were too few recaptures by either genotype to establish significant differences. Conversely, when there was no local retention of seeds, and hence dormancy could not serve a space-holding function, the non-dormant genotype always had a higher probability of recapturing safe sites. These results are consistent with the patterns of conditions favoring dormancy in the previous simulations, showing that dormancy is favored whenever it can serve to hold space. However, these results are confounded by the fact that the favored genotype is more abundant by year 98 of the simulation, and therefore may have a higher probability of recapturing sites simply because there are more seeds of that genotype being dispersed to all sites in the grid. This confounding factor was removed by equalizing the abundances of the two genotypes before tracking

Table 2 Probability of site recapture, by genotype, without equalization of genotype abundances

\begin{tabular}{llll}
\hline \multicolumn{3}{l}{ Fecundity } & \\
\cline { 2 - 4 } & 8 & 32 & 128 \\
\hline Retention & & & \\
0 & (extinct) & $0.573^{*} / 0.164$ & $0.860^{*} / 0.165$ \\
0.2 & $0.034 / 0.408^{*}$ & $0.043 / 0.184^{*}$ & $0.084 / 0.235^{*}$ \\
0.4 & $0.044 / 0.391^{*}$ & $0.032 / 0.198^{*}$ & $0.007 / 0.129 *$ \\
0.6 & $0 / 0.167^{*}$ & $0 / 0.094^{*}$ & $0.014 / 0.115^{*}$ \\
0.8 & $0 / 0.200^{*}$ & $0 / 0.125$ & $0 / 0.059$
\end{tabular}

Note: Probabilities that a site lost by a genotype in year 99 will be recaptured by the nondormant/dormant genotype in year 100 are reported for the nondormant and then the dormant genoptype as a function of fecundity and local retention of seeds. * denote significant differences between genotypes $\left(\chi^{2} \geq 3.841\right.$, df $\left.=1\right)$
Table 3 Probability of site recapture, by genotype, after equalization of genotype abundances

\begin{tabular}{llll}
\hline \multicolumn{3}{l}{ Fecundity } & \\
\cline { 2 - 4 } & 8 & 32 & 128 \\
\hline Retention & & \\
0 & (extinct) & $0.270 / 0.241$ & $0.530^{*} / 0.469$ \\
0.2 & $0.053 / 0.359^{*}$ & $0.086 / 0.211^{*}$ & $0.053 / 0.205^{*}$ \\
0.4 & $0.031 / 0.350^{*}$ & $0.039 / 0.193^{*}$ & $0.033 / 0.194^{*}$ \\
0.6 & $0.025 / 0.169^{*}$ & $0.022 / 0.119^{*}$ & $0.029 / 0.136^{*}$ \\
0.8 & $0 / 0.261 *$ & $0 / 0.107$ & $0 / 0.241^{*}$ \\
\hline
\end{tabular}

Note: Probabilities that a site lost by a genotype in year 49 will be recaptured by the nondormant/dormant genotype in year 50 are reported for the nondormant and then the dormant genoptype as a function of fecundity and local retention of seeds. * denote significant differences between genotypes $\left(\chi^{2} \geq 3.841\right.$, df $\left.=1\right)$

site loss and recovery, and the same patterns hold (Table 3). The dormant genotype is always less successful at recapturing sites when there is no local retention of seeds, whereas the dormant genotype is always more successful at recapturing sites when some seeds are retained locally.

Alternatively, the advantage of dispersal might come from increasing the colonization rate through integrating random dispersal over multiple years (Harper 1977), reducing the probability that an individual site received no seed input. This hypothesis was not supported by additional simulations (Satterthwaite 2004). Although there was no extrinsic environmental variability in these simulations, the stochastic seed rain into individual sites could be considered environmental stochasticity on a very small scale, and dormancy could be selected as a buffer against this stochasticity. This could explain why invasions succeeded over a slightly broader range of parameter space in the stochastic simulations than predicted by the analytic model (compare Figs. 1, 3a). However, in simulations where fractional seeds were allowed and each site received its expected seed input each year, dormancy was still selected for (Satterthwaite 2004), so buffering local scale variability cannot fully explain selection for dormancy in these simulations.

\section{Discussion}

These models demonstrate that selection for seed dormancy does not require externally driven variation 
in the environment or the restrictive assumptions of previous models of sib competition. Specifically, these models suggest that the advantages of dormancy do not depend on high individual fecundity or low relatedness with the offspring of sibs. Thus, the advantage of dormancy should not be reduced by inbreeding, which is common in plants. Further, by comparing the long-term performance of clonal genotypes, this model suggests selection for dormancy that is not subject to parent-offspring conflict and does not depend on maternal control of germination behavior. Therefore, these results suggest that the apparent mismatch between theory and data on how much dormancy plants are predicted to display (Silvertown 1989; Doak et al. 2002; Evans and Dennehy 2005) may be at least partially explained by the importance of dormancy as a space-holding strategy in plant competition.

This mechanism can only be important for plants which compete for a limited number of suitable microsites, however, the current evidence points to at least some degree of safe-site limitation in many plant species (Turnbull et al. 2000). The advantage of dormancy was particularly strong when safe sites were rare ( $s$ small), whereas safe-site limitation may arise from either safe sites being rare or a plant being superabundant and saturating the landscape. It seems appropriate to think that $s$ is not small for plants that form extensive monocultures, but it is likely small for plants that occur at low densities or are patchily distributed. Thus, $s$ is more likely to be small for plants with very specific microhabitat requirements occurring in heterogeneous landscapes, and more likely to be large for microhabitat generalists in homogeneous landscapes.

The degree to which dormancy was favored in these models did depend on the annual survival of dormant seeds, and the simulations presented here assumed a high annual survival of $0.8-0.9$. This is consistent with other models for the evolution of dormancy, which are sensitive to annual seed bank survival (Cohen 1966; Rees and Long 1992; Nilsson et al. 1994). Even the highest survival used in these models implies a seed bank half-life of approximately 6 years. Such seed bank half-lives are not unprecedented, and germination from dormant seeds as old as 10,000 years has been observed (Porsild et al. 1967). Toole and Brown (1946) found that 36 out of 107 species had seeds remaining viable after 39 years, which would require at least $89 \%$ annual survival in those species to retain even $1 \%$ of the original seed bank. In addition, while not all species with seed dormancy have long-lived seed banks (Baskin and Baskin 1998), and surveys of some communities have turned up few species with seeds remaining viable longer than 4 years (Bekker et al. 1997), it is important to note that seed viability is easier to underestimate in the field than it is to overestimate (Bekker et al. 1998).

This model formulation assumed no cost (in terms of reduced total seed production) to produce dormant seeds. Adding a cost to the production of dormant seeds would be functionally equivalent to reducing the survival of dormant seeds, with a resultant restriction in the advantage of dormancy.

Dormancy yields a competitive advantage only when some fraction of each plant's seeds is locally retained. This is nearly universally the case (Harper 1977; Higgins and Richardson 1999), although plants do vary in their dispersal efficiency and more dormancy would be expected in plants with highly restricted dispersal. The proportion of seeds retained in a plant's home site may be quite high-e.g., Bastida and Talavera (2002) found that approximately $80 \%$ of ballistically dispersed seeds were retained under the parent plant's canopy in two species of shrubs.

In reality, dispersal kernels are likely to take on complicated shapes due to plant architecture, a variety of dispersal mechanisms, and environmental influences. Thus, the assumption that seeds either land in a plant's home site or are dispersed uniformly is a gross simplification. Nevertheless, similar qualitative patterns held for more time-consuming simulations using a negative exponential dispersal kernel (Satterthwaite 2004). Further explorations of the impacts of subtly different kernels such as a Weibull could prove interesting, however, it is important to note that in these sorts of spatially explicit, individual-based models the effects of demographic stochasticity can be quite strong and the variance thus created is likely to swamp the effects of subtle changes in dispersal kernels without massive replication. In general, it seems that more realistic dispersal kernels would tend to make it more likely that a plant could colonize nearby sites than distant sites, with the consequence that holding onto nearby sites would be of increased importance. To the extent 
that a locally accumulated seed bank assisted with holding onto these nearby sites, this might increase the selective advantage of dormancy.

The pattern typically seen for plants with dimorphic seeds is consistent with this model (Venable 1989; Olivieri 2001). It is common for plants with dimorphic flowers to produce highly dispersible seeds with little to no dormancy as well as less dispersible seeds that germinate less readily. While this switching between dispersal and dormancy is commonly interpreted to show that the two strategies are alternative means of buffering against environmental variability (Klinkhamer et al. 1987), it is also the expected pattern if dormancy serves as a strategy for spatial competition, since only the less dispersible seeds are likely to perform a space-holding function.

The importance of space-holding strategies in plant competition has long been appreciated, as demonstrated by Lovett Doust's (1981) description of "phalanx" and "guerilla" growth strategies. Perenniality is an obvious alternative strategy for holding space, and thus the observation that longerlived plants tend to be less dormant (Rees 1993) may not be explained entirely by the need for environmental buffering. For annual plants, phylogenetic constraints may make the evolution of increased longevity very costly or even impossible, while adaptations for dormancy may evolve more readily. Dormancy may therefore be the only means for annual plants to move from the "guerilla" to the "phalanx" end of the spectrum in plant competition strategies.

Storage-effect models (Chesson and Warner 1981) have previously demonstrated the importance of holding onto space in interspecific competition. This suggests that dormancy may yield a competitive advantage for plants engaged in interspecific competition for similar safe sites. However, models of plant species coexistence through a competition-colonization tradeoff (Hastings 1980; Tilman 1994) typically neglect seed dormancy (Holmes and Wilson 1998; Levine and Rees 2002; Kidsi and Geritz 2003). Therefore, existing models may err considerably in predicting how easily species can coexist. Dormancy may increase the ability of competitively superior species to hold onto safe sites, such that competitive inferiors are less likely to be able to persist through superior colonization of empty sites. Conversely, environmentally sensitive germination strategies may make it easier for competitively inferior species to persist, as fewer recruits will be lost by germinating into locations where they will be outcompeted.

Acknowledgments This article was improved by comments made by Dan Doak, Marc Mangel, and Ingrid Parker. Support was provided by Environmental Protection Agency STAR graduate research fellowship U-91578601 and a University of California Santa Cruz Chancellor's Fellowship to WHS, a National Science Foundation grant to D. Letourneau, and a CALFED grant to M. Mangel and S. Sogard.

Open Access This article is distributed under the terms of the Creative Commons Attribution Noncommercial License which permits any noncommercial use, distribution, and reproduction in any medium, provided the original author(s) and source are credited.

\section{Appendix}

Invasion by dormant genotype-no seed bank in first year

In this analysis, I relax the assumption that the first invader (dormant genotype) adult to establish is accompanied by dormant siblings in the seed bank. I assume that in year 0 there is a single mutant adult in one site, with no seed bank. The analytic investigation of invasion criteria in this case faces two complications, an expected transient decline in the first year (when the seed bank can make no contribution) and subsequent oscillatory dynamics in the expected size of the seed bank, which will be relatively abundant in year 2 (due to carry-over from seeds produced by the year 0 adult), then smaller in year 3 (due to the temporarily reduced population in year 1 , resulting in few seeds to germinate out of the seedbank in year 3), and so on. Thus, the expected number of sites occupied by the mutant will tend to increase more on odd-year to evenyear transitions than on even-year to odd-year transitions, resulting in damped oscillations around an overall trend of invader increase or decrease. The key question is whether the overall trend in peak (evenyear) expected site occupancy is increasing or decreasing. Since the year 0 population is a special case as the only year in which there can be no seed bank contribution, the best measure of increase or decrease over the short term is $E_{4} / E_{2}$, the ratio between the expected number of mutant-occupied sites in year 4 versus year 2 . 
Fig. 4 Pathways by which seeds produced each year may contribute to the expected number of dormant genotype invaders in subsequent years. Solid lines denote seeds that germinate within a year of release, dashed lines represent seeds that are initially dormant. Note that arrows for seeds dispersing out of the parent's home site are suppressed for transitions starting from year 2 or later to reduce clutter

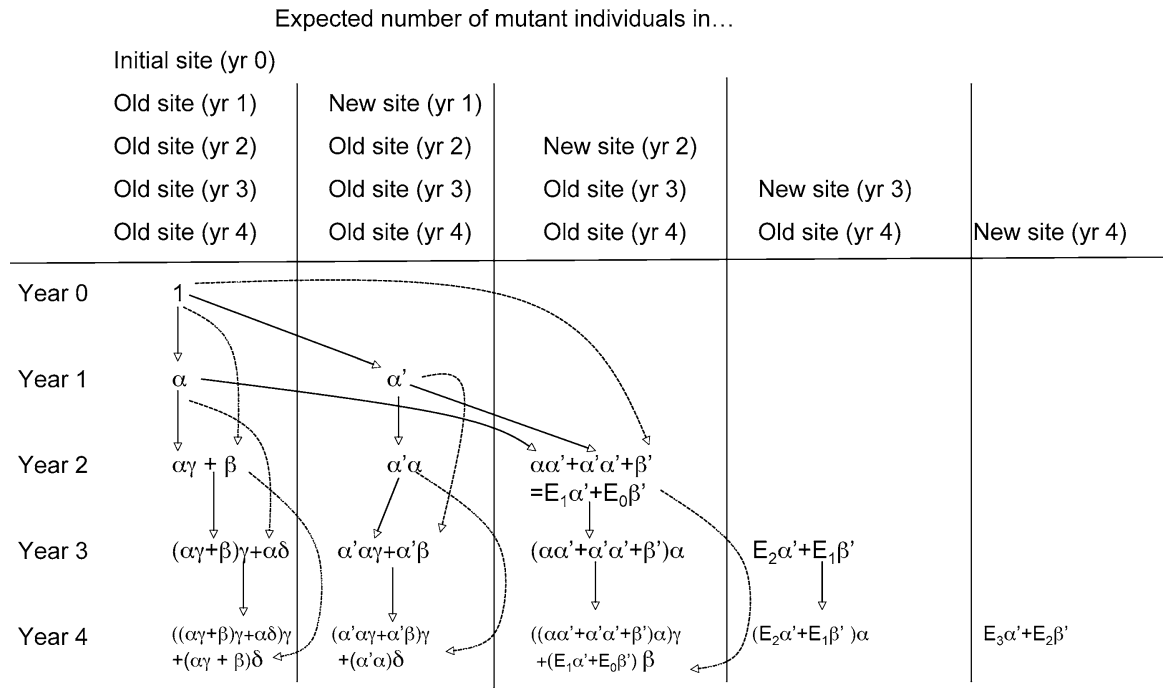

I do not extend this analysis beyond year 4 because the equations become quite cumbersome, and also equations for the probabilities of colonization by different seed types would need to be adjusted as the invader becomes less rare. As currently developed, the model assumes that all seed rain into sites (i.e., all input of seeds not locally produced) was produced by non-dormant adults, and it assumes that every site (including those already captured by mutants) contribute to this seed rain of non-mutant competitors. This assumption makes the analytic model a conservative test for conditions favoring the dormant mutant, since the invader increases in frequency it would face less competition from the non-dormant seed rain.

Let the invasion begin in year 0 with a single adult mutant plant in a single site. Thus, the expected number of sites occupied by mutants in year $0\left(E_{0}\right)$ is 1 . There is no seed bank in year 0 , and no seeds can germinate out of the seed bank in year 1 (instead, seeds produced by the year 0 adult can first germinate in year 2). Figure 4 shows various pathways by which seeds produced each year can contribute to the expected number of sites occupied by invaders in each year.

In year 1 , the expected number of sites occupied by mutants $\left(E_{1}\right)$ is the sum of the probabilities of two independent events:

$\alpha$-the probability that the mutant retains its original site, through a freshly germinating (i.e., never dormant) seed $\alpha$-the probability that the mutant colonizes a new site, through a freshly germinating (i.e., never dormant) seed. Note that such a site becomes an old site from the perspective of the year 2 population, however, no internally produced dormant seeds can germinate out of such sites until year 3, while dormant seeds dispersed from the initial invader may germinate in new sites in year 2. Thus,

$E_{1}=\alpha+\alpha^{\prime}$

In year 2 , there are many contributors to the expected population of mutants, independent chains of events with respective probabilities:

$\alpha \gamma$-The mutant may have retained its old site in year 1 , and retain it again in year 2 through a freshly germinating seed. This probability is not simply $\alpha \alpha$, since in year 2 the freshly produced mutant seeds face competition from previously dormant seeds as well as germinating seeds produced onsite and from the seed rain. $\gamma$ is less than $\alpha$, as shown below.

$\beta$ - The mutant may colonize the original (year 0 ) home site through a dormant seed produced by the year 0 adult. As described below, $\beta$ is the sum of two independent joint probabilities - the mutant may have retained the site in year 1 and retained it in year 2 through a dormant seed germinating out of the seed bank, or it may have lost the site in year 1 and recaptured it in year 2 through a dormant seed germinating out of the seed bank. 
$\alpha^{\prime} \alpha-A$ site newly captured in year 1 may be retained in year 2 by a freshly produced mutant seed produced by the new mutant occupant of that site.

$\alpha \alpha^{\prime}$ - The mutant may retain its original site in year 1 , and colonize a new site in year 2 through a freshly produced germinating seed dispersed out of the home site.

$\alpha^{\prime} \alpha^{\prime}$-The mutant may have captured a new site in year 1 and dispersed a seed from that site which captures a new site in year 2 .

$\beta$ '-A dormant seed dispersed by the original invader (the adult from year 0 ) may germinate out of the seed bank and colonize a new site in year 2 .

Thus, the expected number of sites occupied by mutants in year 2 is:

$N_{2}=\alpha \gamma+\beta+\alpha^{\prime} \alpha+\alpha \alpha^{\prime}+\alpha^{\prime} \alpha^{\prime}+\beta^{\prime}$

Using in variables as defined in the main text, these components can be calculated as follows:

$\alpha=\frac{g r F}{g r F+s N(1-r) F \frac{1}{N}}=\frac{g r F}{g r F+s(1-r) F}$

That is, the total number of germinating mutant seeds retained in the mutant's home site divided by the total number of germinating seeds in the mutant's home site (the mutant's retained seeds plus seed rain from resident wild-type plants in other sites). Given $N$ total sites, there will be $s N$ wild-type plants each producing $F$ seeds of which fraction $(1-r)$ disperse, landing in the mutant's home site with probability $1 /$ $N$. Note that this calculation implicitly assumes that even the invader's original home site contributes to the wild-type seed rain, penalizing the invader and making this a conservative test of the advantage of dormancy.

$\alpha^{\prime}=\frac{s(1-r) g F}{r F+s(1-r) F}$

That is, the mutant disperses $(1-r) g F$ germinating seeds, of which a fraction $s$ will land in safe sites. Assuming that the number of sites is large, only one mutant seed will land in each (new) site, establishing with a probability inversely proportional to the total number of seeds in that site $-r F$ retained seeds produced by its wild-type resident and $s(1-r) F$ wildtype seed rain as described earlier. The mutant seed is not explicitly included in the denominator, however, as noted earlier this calculation of wild-type seed rain assumes that every site (including the invader's original home site) contains a wild-type individual, making this a conservative test of the advantage of dormancy.

$\gamma=\frac{g r F}{(1-g) b r F+g r F+s(1-r) F}$

For freshly produced seeds germinating in year 2 in a site retained by the invader from year 0 to year 1 , the total pool of competitors includes previously dormant seeds from the seed bank $((1-g) b r F)$ along with retained freshly produced germinating seeds and the wild-type seed rain as in the denominator for $\alpha$. Thus $\gamma$ is less than $\alpha$.

$$
\begin{aligned}
\beta= & \alpha \frac{(1-g) b r F}{g r F+(1-g) b r F+s(1-r) F} \\
& +(1-\alpha) \frac{(1-g) b r F}{r F+(1-g) b r F+s(1-r) F}
\end{aligned}
$$

The first term in the sum above represents the joint probability that the invader retains its home site in year $1(\alpha)$ and that a dormant seed originally produced in year 0 captures the site in year 2 , given the expected total-germinating seed input into the site if it was occupied by a mutant during year 1 ( $g r F$ freshly produced germinating mutant seeds, $(1-$ $g) b r F$ seeds out of the seed bank, and a wild-type seed rain as before). The second term represents the joint probability that the invader failed to retain its home site in year $1(1-\alpha)$ and that a dormant seed originally produced in year 0 captures the site in year 2 , given the expected total germinating seed input into the site if it was occupied by a wild-type plant in year 1 ( $r F$ seeds from the wild-type resident, $(1-$ $g) b r F$ seeds germinating out of the seed bank, and a wild-type seed rain as before).

$\beta^{\prime}=\frac{s(1-r)(1-g) b F}{r F+s(1-r) F}$

That is, $(1-r)(1-g) F$ dormant seeds are dispersed in year 0 , with fraction $s$ reaching safe sites and fraction $b$ surviving to germinate in year 2 . The denominator is the same as for the calculation of $\alpha^{\prime}$.

Substituting these values (noting that $F$ cancels out of every term) into

$E_{1}=\alpha+\alpha^{\prime}$

and 


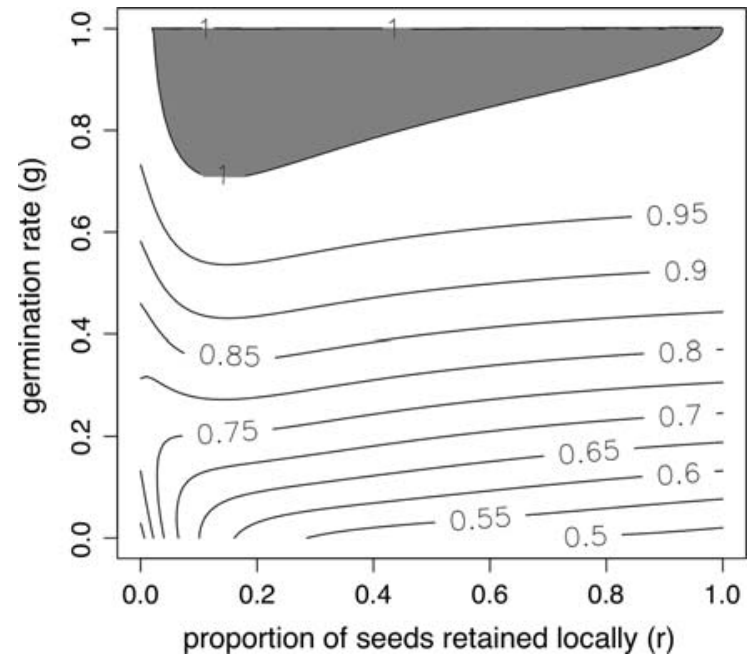

Fig. 5 The expected ratio of year 4:year 2 number of occupied sites for an invading dormant genotype given annual seed bank survival equal to 0.9 and $5 \%$ of sites safe. The shaded region indicates expected invasion success

$E_{2}=\alpha \gamma+\beta+\alpha^{\prime} \alpha+\alpha \alpha^{\prime}+\alpha^{\prime} \alpha^{\prime}+\beta^{\prime}$

As noted previously, $E_{2}$ alone is not a good metric of invasion success, since there must be a transient decline in invader abundance in year 1 even for successful invaders. $E_{2} / E_{0}$ is a poor metric for the same reason, while $E_{2} / E_{1}$ is a poor metric of invasion success due to the oscillatory dynamics introduced by the transient decline, such that the best estimates of invader growth rate come from comparing expected numbers of sites over 2 year intervals. Thus, the calculations must be extended to $E_{3}$ and $E_{4}$.

The calculations can be extended to years 3 and 4 with one additional term. The probability of the original home site being captured by a dormant seed in year 3 becomes $\alpha \delta$ (Fig. 4). The $\alpha$ term represents the probability that there was a mutant plant present in the site in year 1 to leave dormant seeds to germinate in year 3 . Given that, the previously used $\beta$ term must be adjusted to $\delta$ based on new probabilities for the occupant in year 2 being a mutant, as follows ( $\gamma$ replaces $\alpha$ where it occurs in the original equation for $\beta$ ):

$$
\begin{aligned}
\delta= & \gamma \frac{(1-g) b r F}{g r F+(1-g) b r F+s(1-r) F} \\
& +(1-\gamma) \frac{(1-g) b r F}{r F+(1-g) b r F+s(1-r) F}
\end{aligned}
$$

If a single mutant adult has probability $\alpha^{\prime}$ of colonizing a new site through a freshly produced

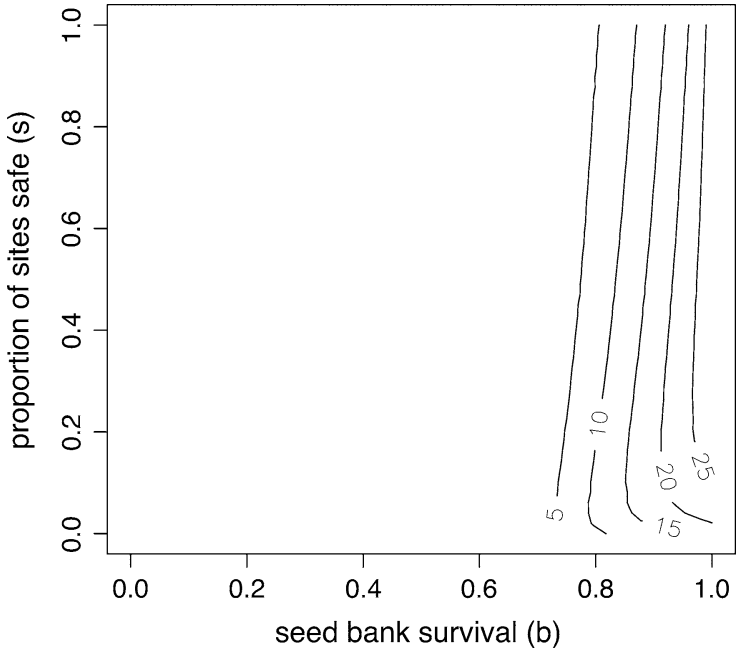

Fig. 6 The percent of germination-seed retention phase space over which the dormant genotype can invade, for different combinations of local seed retention and safe site availability

dispersing seed, the total number of such events expected in year $y$ is $N_{y}-{ }_{1} \alpha^{\prime}$. Likewise, if a single mutant adult has probability $\beta$ ' of colonizing a new site (two years in the future) through a dispersed dormant seed, the total number of such events expected in year $y$ is $N_{y}-{ }_{2} \beta$ '.

Note also that every term including a $\beta$ or $\delta$ term represents a pathway available to the dormant mutant but not the non-dormant resident.

Thus, the total number of sites occupied by invaders expected in year 3 is:

$$
\begin{aligned}
E_{3}= & (\alpha \gamma+\beta) \gamma+\alpha \delta+\alpha^{\prime} \alpha \gamma+\alpha^{\prime} \beta \\
& +\left(\alpha \alpha^{\prime}+\alpha^{\prime} \alpha^{\prime}+\beta^{\prime}\right) \alpha+E_{2} \alpha^{\prime}+E_{1} \beta^{\prime}
\end{aligned}
$$

And the expected number of sites occupied by invaders in year 4 is:

$$
\begin{aligned}
E_{4}= & ((\alpha \gamma+\beta) \gamma+\alpha \delta) \gamma+(\alpha \gamma+\beta) \delta+\left(\alpha^{\prime} \alpha \gamma+\alpha^{\prime} \beta\right) \gamma \\
& +\left(\alpha^{\prime} \alpha\right) \delta+\left(\left(\alpha \alpha^{\prime}+\alpha^{\prime} \alpha^{\prime}+\beta^{\prime}\right) \alpha\right) \gamma \\
& +\left(E_{1} \alpha^{\prime}+E_{0} \beta^{\prime}\right) \beta+\left(E_{2} \alpha^{\prime}+E_{1} \beta^{\prime}\right) \alpha \\
& +E_{3} \alpha^{\prime}+E_{2} \beta^{\prime}
\end{aligned}
$$

As noted earlier, due to oscillatory dynamics created by the seed bank, the population of invaders suffers a transient decline in year 1 and is high on even years and low on odd years. Therefore, the most appropriate measure of invader growth rate is $E_{4} / E_{2}$. Calculating this ratio for various combinations of local retention $(r)$ and germination $(g)$, identifies the 
conditions under which a dormant mutant can invade (first for $b=0.9$ and proportion of sites safe $s=0.05$, Fig. 5, compare with Fig. 1). As before, local retention of seeds tends to favor invasion, but genotypes with low and intermediate germination rates are generally not able to invade.

Thus, an invader with germination rates near (but less than) 1 can increase when rare. Invasion is easier when local seed retention is fairly low, but becomes more difficult at the lowest levels of seed retention. In such cases, colonization of a plant's home site is still the most likely way for an individual to replace itself (since few dispersing seeds find safe sites, and face many non-sib competitors when they do) but there is more risk of losing the home site, making the ability to recapture a home site through the seed bank more advantageous. Very high seed retention may also hinder invasion because very few new sites are ever colonized. If seed retention drops to very low values, the home site is no longer the easiest site to colonize, and this advantage disappears.

We can determine the importance of seed bank survival and safe site availability in determining the fitness of the dormant strategy by calculating the proportion of $g-r$ phase space for which invasion succeeds (i.e., $E_{\downarrow} / E_{2}>1$ ), across different values for $b$ and $s$ (Fig. 6, compare with Fig. 2). This indicates that seed bank survivals above approximately 0.7 are required for the dormant genotype to invade. The proportion of safe sites has less of an effect, although invasions are less likely to succeed as more sites are safe, or if safe sites are very rare.

Resistance against invasion for the dormant genotype once established

In order to show that once established, the dormant genotype can resist invasion, I consider invasion of a non-dormant mutant into a saturated population of plants with the dormant genotype. Thus, in year 0 the non-dormant invader occupies a single site, there is, however, a seed bank in that site left over from the dormant occupant the year before the invasion began.

The non-dormant invader can replace itself in its original site (with probability $a$ ), or colonize a new site (with probability $a^{\prime}$ ). Once the non-dormant invader has occupied a site for consecutive years, it eliminates the locally retained seed bank of that site (although not dormant seeds dispersing in), thus its probability of retaining that site shifts to $c$. Therefore, the expected number of sites occupied through time by the nondormant invader can be calculated as follows: Individual components of this calculation are as follows: The nondormant invader retains $r F$ seeds in its home site, which therefore compete against the $r F$ retained seeds of the invader, $b(1-g) r F$ seeds from the seed bank produced by the occupant the previous year, $s g(1-r) F$ freshly germinating seeds from the resident population seed rain, and $s b(1-$ $g)(1-r) F$ seeds from the seed bank produced by the resident population seed rain the previous year.

$a^{\prime}=\frac{s(1-r) F}{g r F+b(1-g) r F+s g(1-r) F+s b(1-g)(1-r) F}$

The non-dormant invader disperses $(1-r) F$ seeds of which a fraction $s$ reach safe sites occupied by the dormant genotype resident. These sites will have received $g r F$ retained freshly germinating seeds produced by the dormant-genotype occupant, $b(1-g) r F$ seeds from the seed bank produced by the dormant genotype occupant the previous year, $s g(1-r) F$ freshly germinating seeds from the resident population seed rain, and $s b(1-g)(1-r) F$ seeds from the seed bank produced by the resident population seed rain the previous year. As in previous calculations, the one invader seed does not enter into the denominator, since the seed rain already assumes the contribution of seeds produced by a dormant-genotype adult in every site. However, in this case dormant genotype adults disperse fewer freshly germinating seeds than the invader, thus this equation slightly underestimates the number of competitors faced by the non-dormant invader's seed. Thus, this is a conservative test of the ability of the dormant genotype to resist invasion.

$c=\frac{r F}{r F+s g(1-r) F+s b(1-g)(1-r) F}$

Once the non-dormant invader has retained a site for consecutive years, the $b(1-g) r F$ locally produced seed bank is eliminated, removing one term from the denominator as compared to $a$.

As with the dormant invader, the non-dormant invader faces a transient decline in the expected number of occupied sites for year 1 , since in year 0 only the non-dormant invader has not displaced any residents long enough to eliminate the resident seed 
Fig. 7 The expected ratio of year 3:year 2 (a) or year 4:year 3 (b) number of occupied sites for an invading non-dormant genotype given annual seed bank survival equal to 0.9 for the resident and $5 \%$ of sites safe
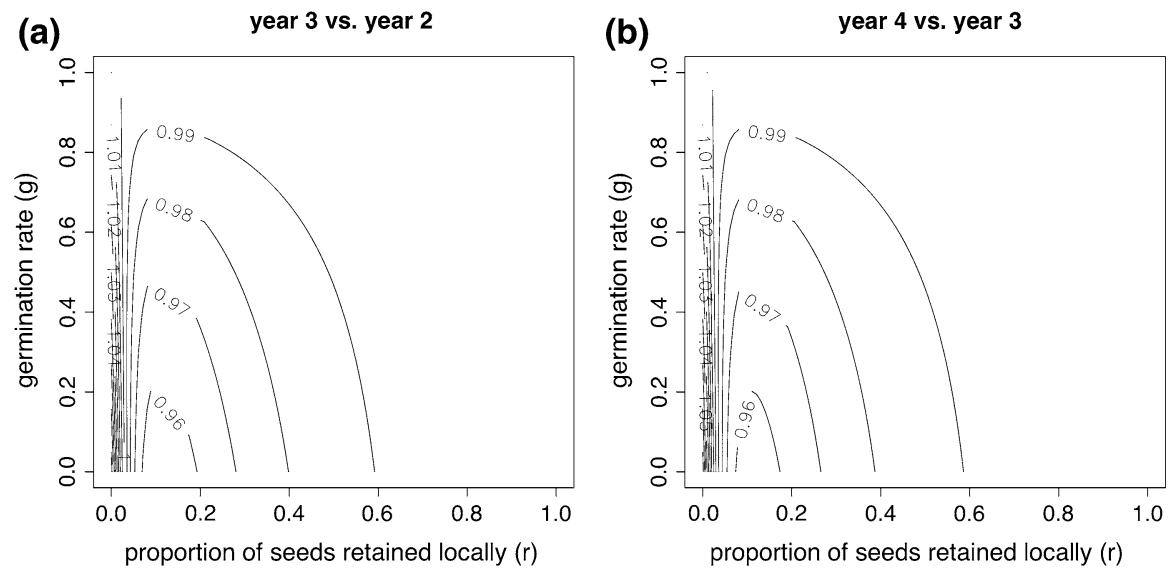

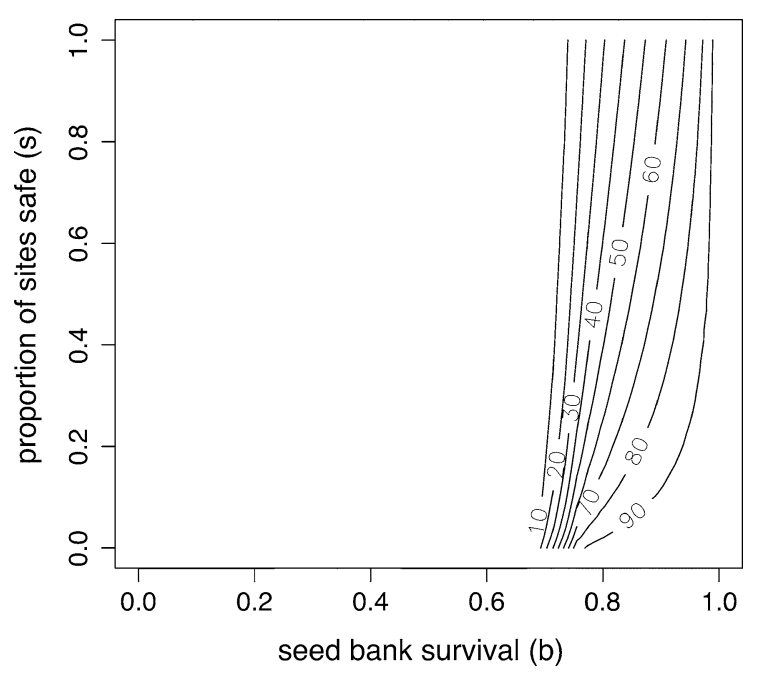

Fig. 8 The percent of germination-seed retention phase space over which the dormant genotype can resist invasion by a nondormant invader, for different combinations of local seed retention and safe site availability

bank at its home site. Thus, $E_{1} / E_{0}$ is an overly pessimistic estimate of non-dormant invader success, and $E_{2} / E_{1}$ is overly optimistic because it partially reflects rebound from the low expected number of sites occupied in year 1 . However, $E_{3} / E_{2}$ and $E_{4} / E_{3}$ provide good and convergent assessments of invader success, and show that the non-dormant invader can only succeed for regions of parameter space where the dormant genotype invader could not invade. In fact, invasion of the non-dormant invader fails over a broader range of parameter space than that over which invasion of the dormant invader succeeds, similar to the simulation results (Fig. 7, compare with Fig. 5, note that invasion by the non-dormant genotype succeeds only when seed retention $r$ is very low). Note that $g=1$ always leads to the non-dormant invader exactly replacing itself on average, since there is no difference between the invader and resident. Also, $r=1$ leads to the invader exactly replacing itself (after year 1) since the occupant of any site always retains it if no seeds disperse outside the parent site.

Dormant seed survival and safe site availability will also affect the ability of the dormant genotype to resist invasion, so I also calculated the percent of $g-$ $r$ phase space over which the dormant genotype can resist invasion over a range of $b$ and $s$ values. Although the seed bank survival required to resist invasion is similar to that required to invade, again the dormant genotype can resist invasion for a wider range of $g$ and $r$ parameter values than it can invade over (Fig. 8, compare with Fig. 6).

\section{References}

Baskin CC, Baskin JM (1998) Seeds: ecology, biogeography and evolution of dormancy and germination. Academic Press, San Diego, CA

Bastida F, Talavera S (2002) Temporal and spatial patterns of seed dispersal in two Cistus species. Ann Bot 89:427-434

Bekker RM, Verweij GL, Smith REN, Renie R, Bakker JP, Sneider S (1997) Soil seed banks in European grasslands: does land use affect regeneration perspectives? J Appl Ecol 34:1293-1310

Bekker RM, Bakker JP, Grandin U, Kalamees R, Milberg P, Poschold P, Thompson K, Willems JH (1998) Seed size, shape and vertical distribution in the soil: indicators of seed longevity. Funct Ecol 12:834-842

Cheplick GP (1992) Sibling competition in plants. J Ecol 80:567-575 
Chesson PL, Warner R (1981) Environmental variability promotes coexistence in lottery competitive systems. Am Nat 117:923-943

Clauss MJ, Venable DL (2000) Seed germination in desert annuals: an empirical test of adaptive bet-hedging. Am Nat 155:168-186

Cohen D (1966) Optimizing reproduction in a randomly varying environment. J Theor Biol 12:119-129

Doak DF, Thomson DM, Jules ES (2002) PVA for plants: understanding the demographic consequences of seed banks for population health. In: Beissinger S, McCullough D (eds) Population viability analysis. University of Chicago Press, Chicago, pp 312-337

Ehrman T, Cocks PS (1996) Reproductive patterns in annual legume species on an aridity gradient. Vegetatio 122:47-59

Ellner S (1986) Germination dimorphisms and parent-offspring conflict in seed germination. J Theor Biol 123:173-185

Ellner S (1987) Competition and dormancy: a reanalysis and review. Am Nat 130:798-803

Evans MEK, Dennehy JJ (2005) Germ banking: bet-hedging and variable release from egg and seed dormancy. Q Rev Biol 80:431-451

Evans MEK, Ferriere R, Kane MJ, Venable DL (2007) Bet hedging via seed banking in desert evening primroses (Oenothera, Onagraceae): demographic evidence from natural populations. Am Nat 169:184-194

Gillespie JH (1977) Natural selection for variances in offspring numbers: a new evolutionary principle. Am Nat 111:1010-1014

Gutterman Y, Edine L (1988) Variations in seed germination of Helianthemum vescarium and $H$. ventosum seeds from two different altitudes in the Negev Highlands, Israel. J Arid Environ 15:261-267

Hacker JB, Ratcliff D (1989) Seed dormancy and factors controlling dormancy breakdown in buffel grass accessions from contrasting provenances. J Appl Ecol 26:201-212

Hamilton WD (1967) Extraordinary sex ratios. Science 156:477-488

Harper JL (1977) The population biology of plants. Academic Press, London

Hastings A (1980) Disturbance, coexistence, history and competition for space. Theor Popul Biol 18:363-373

Higgins SI, Richardson DM (1999) Predicting plant migration rates in a changing world: the role of long-distance dispersal. Am Nat 153:464-475

Holmes EE, Wilson HB (1998) Running from trouble: longdistance dispersal and the competitive coexistence of inferior species. Am Nat 151:578-586

Hyatt LA, Evans AS (1998) Is decreased germination fraction associated with risk of sibling competition? Oikos 83:29-35

Jain SK (1982) Variation and adaptive role of seed dormancy in some annual grassland species. Bot Gazette 143:101-106

Kidsi E, Geritz SAH (2003) On the coexistence of perennial plants by the competition-colonization tradeoff. Am Nat 161:350-354

Klinkhamer PGL, DeJong TJ, Metz JAJ, Val J (1987) Life history tactics of annual organisms: the joint effects of dispersal and delayed germination. Theor Popul Biol 32:127-156

Kobayashi Y, Yamamura N (2000) Evolution of seed dormancy due to sib competition: effect of dispersal and inbreeding. J Theor Biol 202:11-24
Lalonde RG, Roitberg BD (2006) Chaotic dynamics can select for long-term dormancy. Am Nat 168:127-131

Leck MA, Parker VT, Simpson RL (1989) Ecology of soil seed banks. Academic Press, London

Levine JM, Rees M (2002) Coexistence and relative abundance in annual plant assemblages: The roles of competition and colonization. Am Nat 160:452-467

Lovett Doust L (1981) Intraclonal variation and competition in Ranunculus repens. New Phytol 89:495-502

Nilsson P, Fagerstrom T, Tuomi J, Astrom M (1994) Does seed dormancy benefit the mother plant by reducing sib competition? Evol Ecol 8:422-430

Olivieri I (2001) The evolution of seed heteromorphism in a metapopulation: interactions between dispersal and dormancy. In: Silvertown J, Antonovics J (eds) Integrating ecology and evolution in a spatial context. Blackwell Scienctific, Oxford, pp 245-268

Petru M, Tielborger K (2008) Germination behavior of annual plants under changing climatic conditions: separating local and regional environmental effects. Oecologia 155:717-728

Philippi T (1993a) Bet-hedging germination of desert annuals: beyond the first year. Am Nat 142:474-487

Philippi T (1993b) Bet-hedging germination of desert annuals: variation among populations and maternal affects in Lepidium lasiocarpum. Am Nat 142:488-507

Platenkamp GAJ (1991) Phenotypic plasticity and population differentiation in seeds and seedlings of the grass $A n$ thoxanthum odoratum. Oecologia 88:515-520

Porsild AE, Harrington CR, Mulligan GA (1967) Lupinus arcticus var Wats. grown from seeds of Pleistocene age. Science 158:113-114

R Development Core Team (2007) R: a language and environment for statistical computing. R Foundation for Statistical Computing, Vienna, Austria. ISBN 3-900051-070, URL http://www.R-project.org

Rees M (1993) Trade-offs among dispersal strategies in British plants. Nature 366:150-152

Rees M (1994) Delayed germination of seeds: a look at the effects of adult longevity, the timing of reproduction and population age/stage structure. Am Nat 144:43-64

Rees M, Crawley MJ (1991) Do plant populations cycle. Funct Ecol 5:580-582

Rees M, Long MJ (1992) Germination biology and the ecology of annual plants. Am Nat 139:484-508

Satterthwaite WH (2004) Dispersal in space and time and its importance to plant population dynamics. Ph.D thesis, University of California at Santa Cruz, p 154

Satterthwaite WH (2007) The importance of dispersal in determining seed versus safe site limitation of plant populations. Plant Ecol 193:113-130

Shem-Tov S, Zaady E, Gutterman Y (2002) Germination of Carrichtera annua (Brassicaceae) seeds on soil samples collected along a rainfall gradient in the Negev Desert of Israel. Israel J Plant Sci 50:113-118

Silvertown L (1989) The demographic and evolutionary consequences of seed dormancy. In: Davey AJ, Hutchings MJ, Watkinson AR (eds) Plant population ecology. Blackwell Scientific, Oxford, pp 205-219

Slatkin M (1974) Hedging one's evolutionary bets. Nature 250:704-705 
Tielborger K, Valleriani A (2005) Can seeds predict their future? Germination strategies of density-regulated desert annuals. Oikos 111:235-244

Tilman D (1994) Competition and biodiversity in spatially structured habitats. Ecology 75:2-16

Toole EH, Brown E (1946) Final results of the Duvel buried seed experiment. J Agric Res 72:201-210

Turnbull LA, Crawley MJ, Rees M (2000) Are plant populations seed-limited? A review of seed sowing experiments. Oikos 88:225-238
Venable DL (1989) Modeling the evolutionary ecology of seed banks. In: Leck MA, Parker VT, Simpson RL (eds) Ecology of soil seed banks. Academic Press, New York, pp 67-90

Venable DL, Lawlor L (1980) Delayed germination and dispersal in desert annuals: escape in space and time. Oecologia 46:272-282

Zammit C, Zedler PH (1990) Seed yield, seed size and germination behaviour in the annual Pogogyne abramsii. Oecologia 84:24-28 